\title{
Context integration in visual processing: a computational model of center-surround suppression in the visual system
}

\author{
Christoph Metzner $^{1,2^{*}}$, Achim Schweikard ${ }^{1}$, Bartosz Zurowski ${ }^{3}$ \\ From Nineteenth Annual Computational Neuroscience Meeting: CNS*2010 \\ San Antonio, TX, USA. 24-30 July 2010
}

A dysfunction of GABAergic neurotransmission is hypothesized to be an important factor in the pathophysiology of schizophrenia [1], depression and anxiety disorders. Findings of decreased center-surround suppression (CSS, i.e. the mutual inhibition of a focal visual stimulus and its surrounding) have been interpreted in terms of GABAergic dysfunction [2]. Consistently, strongly decreased CSS is reported in schizophrenic patients [3]. However, the underlying mechanisms of this decrease remain unclear.

\section{Methods}

We developed a biologically inspired neural network model of the human primary visual system, consisting of two retinas, two layers of dorso-lateral geniculate nucleus and section $B$ and $C$ of layer 4 of the primary visual cortex. The model was built using GENESIS (www.genesis-sim.org) and comprises about 150,000 neurons. Three different kinds of neurons were incorporated, namely thalamic relay neurons, and excitatory and inhibitory cortical neurons. The processing of input in the retina was simply modeled as random-spike units, where the average firing rate corresponds to the input intensity. The neurons consisted of a soma, an axon and two or four dendrites for relay or inhibitory and excitatory neurons, respectively. We used different ionic channels to reproduce the desired firing behaviour. Synaptic transmission was implemented using the built-in excitatory and inhibitory synaptic channels.

\section{Results}

We designed a protocol to explore the center-surround suppression capabilities of our model. The retina was

\footnotetext{
* Correspondence: metzner@rob.uni-luebeck.de

'Institute for Robotics and Cognitive Systems, University of Luebeck, 23538 Luebeck, Schleswig-Holstein, Germany
}

stimulated with contrast stimuli similar to those used in [2]. We compared activity in central neurons of layer $4 \mathrm{~B}$ and $4 \mathrm{C}$ in response to two stimuli with a focal circle of $40 \%$ Michelson contrast, where the target stimulus had a high-contrast surrounding while the reference had a uniform surrounding. We found a decrease in activity of $27.73 \%$ in layer $4 \mathrm{~B}$ and one of $31.41 \%$ layer $4 \mathrm{C}$ when comparing responses to the target with responses to the reference.

A plausible neural network model of the human primary visual system which is consistent with psychophysical effects previously reported in humans [2] is a starting point to further investigate the role of GABAergic inhibition in visual context integration. The capabilities of the model to explain both individual variance of CSS strength and CSS in pathological conditions of GABAergic neurotransmission remains to be tested. We plan to do so on the basis of psychophysical CSS data in humans and corresponding in vivo measurements of GABA and Glutamate concentrations in primary visual cortex using Proton Magnetic Resonance Spectroscopy (1H-MRS).

\section{Author details}

'Institute for Robotics and Cognitive Systems, University of Luebeck, 23538 Luebeck, Schleswig-Holstein, Germany. ${ }^{2}$ Graduate School for Computing in Medicine and Life Sciences,University of Luebeck, 23538 Luebeck, SchleswigHolstein, Germany. ${ }^{3}$ Department of Psychiatry, University of Luebeck, 23538 Luebeck, Schleswig-Holstein, Germany.

Published: 20 July 2010

\section{References}

1. Wassef A, Baker J, Kochan LD: GABA and schizophrenia: A review on basic science and clinical studies. J Clin Psychopharmacol 2003, 23(6):601-640. 
2. Golomb JD, McDavitt JRB, Ruf BM, Chen Jl, Saricicek A, Maloney KH, Hu J, Chun MM, Bhagwagar Z: Enhanced visual motion perception in major depressive disorder. J Neurosci 2009, 29(28):9072-9077.

3. Dakin S, Carlin P, Hemsley D: Weak suppression of visual context in chronic schiophrenia. Curr Biol 2005, 15(20):R822-R824.

doi:10.1186/1471-2202-11-S1-P100

Cite this article as: Metzner et al:: Context integration in visual

processing: a computational model of center-surround suppression in the visual system. BMC Neuroscience 2010 11(Suppl 1):P100.

Submit your next manuscript to BioMed Central and take full advantage of:

- Convenient online submission

- Thorough peer review

- No space constraints or color figure charges

- Immediate publication on acceptance

- Inclusion in PubMed, CAS, Scopus and Google Scholar

- Research which is freely available for redistribution

Submit your manuscript at www.biomedcentral.com/submit
C Biomed Central 